\title{
Constructing non-orthogonal split-split-plot designs using some resolvable block designs
}

\author{
Iwona Mejza ${ }^{1}$, Katarzyna Ambroży-Deręgowska ${ }^{1}$, Kazuhiro Ozawa ${ }^{2}$, \\ Stanisław Mejza ${ }^{1}$, Shinji Kuriki ${ }^{3}$ \\ ${ }^{1}$ Department of Mathematical and Statistical Methods, Poznań University of Life Sciences, \\ Poznań, Poland, e-mail: iwona.mejza@up.poznan.pl \\ ${ }^{2}$ Department of Nursing, Gifu College of Nursing, Gifu, Japan \\ ${ }^{3}$ Department of Mathematical Sciences, Graduate School of Engineering, Osaka Prefecture \\ University, Osaka, Japan
}

\begin{abstract}
SUMMARY
We consider a new method of constructing non-orthogonal (incomplete) split-split-plot designs (SSPDs) for three $(A, B, C)$ factor experiments. The final design is generated by some resolvable incomplete block design (for the factor $A$ ) and by square lattice designs for factors $B$ and $C$ using a modified Kronecker product of those designs (incidence matrices). Statistical properties of the constructed designs are investigated under a randomized-derived linear model. This model is strictly connected with a four-step randomization of units (blocks, whole plots, subplots, sub-subplots inside each block). The final SSPD has orthogonal block structure (OBS) and satisfies the general balance (GB) property. The statistical analysis of experiments performed in the SSPD is based on the analysis of variance often used for multistratum experiments. We characterize the SSPD with respect to the stratum efficiency factors for the basic estimable treatment contrasts. The structures of the vectors defining treatment contrasts are also given.
\end{abstract}

Key words: General balance property, Efficiency factors, Incomplete split-split-plot designs, Khatri-Rao product of matrices, Resolvable designs

\section{Introduction}

The main purpose of this paper is to provide a new method of constructing nonorthogonal (incomplete) split-split-plot designs (SSPDs) for experiments with three or more factors. To simplify the discussion, our considerations will be limited to three factors only. The traditional SSPD applied to three-factor experiments commonly used in agricultural research is an extension of a split- 
plot design serving to accommodate the third factor. In practice, it is mostly applied to orthogonal (complete) experiments (by which we mean here that all treatment combinations occur once in each block); see e.g. Gomez and Gomez (1984). Giving up the requirement of completeness of the SSPD allows the use of larger numbers of levels of the factors (treatments). Generally, in constructions of incomplete designs the ordinary Kronecker product of matrices is often used. Then different experimental designs from the rich class of block designs (orthogonal, non-orthogonal balanced, partially balanced designs, etc.) are usually taken into account as seed designs to generate a final SSPD (e.g. Mejza, 1997a, 1997b; Ambroży and Mejza, 2011, 2012, 2013). Such designs indeed have many interesting statistical properties; they make it possible, for instance, to use control treatments with a different number of replicates than other treatments, but then the experiments are quite large.

In this paper we propose a new construction of an incomplete SSPD with a smaller number of experimental units, using the Khatri-Rao product (also called semi-Kronecker product) of matrices, which was defined by Khatri and Rao (1968) as a modification of the ordinary Kronecker product (see also Rao and Mitra, 1971; Gupta and Mukerjee, 1989).

The considerations are based on a linear model of observations, called a randomized-derived model (e.g. Mejza, 1997a; Ambroży and Mejza, 2011, 2013). We will limit our considerations to an incomplete SSPD with orthogonal block structure (OBS) and the general balance (GB) property (see e.g. Mejza, 1992). These statistical properties of the considered design allow one to apply Nelder's approach to the analysis of variance for multistratum experiments (Nelder, 1965a, 1965b; Houtman and Speed, 1983). The analysis is presented in terms of basic contrasts, introduced by Pearce et al. (1974).

The paper can be treated as a generalization of some earlier ideas on the investigation of statistical properties and constructions of incomplete split-plot designs (cf. for example Mejza and Mejza, 1984; Mejza et al., 2001; Ozawa et al., 2004, 2018; Kuriki and Nakajima, 2007) for three-factor designs of split-plot type. 


\section{Linear model}

Let us consider a three-factor experiment in which the first factor, say $A$, has $v_{1}$ levels (also called the whole plot treatments or $A$ treatments), the second factor, say $B$, has $v_{2}$ levels (called the subplot treatments or $B$ treatments), and the third factor, say $C$, has $v_{3}$ levels (called the sub-subplot treatments or $C$ treatments). Thus $v=v_{1} v_{2} v_{3}$ denotes the number of all treatment combinations in the experiment. It is assumed that the experimental material can be divided into $b$ blocks with $k_{1}\left(<v_{1}\right)$ whole plots. Then each whole plot is divided into $k_{2}$ $\left(<v_{2}\right)$ subplots with $k_{3}\left(<v_{3}\right)$ sub-subplots. The blocks, the whole plots, the subplots and the sub-subplots are independently randomized before conducting the experiment. As a result of certain assumptions and four independent randomization processes in the experiment, the mixed linear model of the vector $\boldsymbol{y}$ of $n\left(=b k_{1} k_{2} k_{3}\right)$ observations has the form:

$$
\boldsymbol{y}=\boldsymbol{\Delta}^{\prime} \boldsymbol{\tau}+\sum_{f=1}^{4} \boldsymbol{D}_{f}^{\prime} \boldsymbol{\eta}_{f}+\boldsymbol{e},
$$

with the following properties:

$$
E(\boldsymbol{y})=\boldsymbol{\Delta}^{\prime} \boldsymbol{\tau} \quad \text { and } \quad \operatorname{Cov}(\boldsymbol{y})=\sum_{f=1}^{4} \boldsymbol{D}_{f}^{\prime} \boldsymbol{V}_{f} \boldsymbol{D}_{f}+\sigma_{e}^{2} \boldsymbol{I}_{n},
$$

where $\boldsymbol{\Delta}^{\prime}$ is a known design matrix for $v$ treatment combinations, $\boldsymbol{\tau}(v \times 1)$ is the vector of fixed treatment combination effects, and $\boldsymbol{D}_{1}^{\prime}, \boldsymbol{D}_{2}^{\prime}, \boldsymbol{D}_{3}^{\prime}, \boldsymbol{D}_{4}^{\prime}$ are respectively $(n \times b),\left(n \times b k_{1}\right),\left(n \times b k_{1} k_{2}\right),\left(n \times b k_{1} k_{2} k_{3}\right)$ design matrices for blocks, the whole plots (within the blocks), the subplots (within the whole plots inside the blocks), and the sub-subplots (within the subplots inside the whole plots and blocks). They are expressed by: $D^{\prime}{ }_{1}=\boldsymbol{I}_{b} \otimes \mathbf{1}_{k_{1}} \otimes \mathbf{1}_{k_{2}} \otimes \mathbf{1}_{k_{3}}, \boldsymbol{D}^{\prime}{ }_{2}=\boldsymbol{I}_{b} \otimes$ $\boldsymbol{I}_{k_{1}} \otimes \mathbf{1}_{k_{2}} \otimes \mathbf{1}_{k_{3}}, \quad D^{\prime}{ }_{3}=\boldsymbol{I}_{b} \otimes \boldsymbol{I}_{k_{1}} \otimes \boldsymbol{I}_{k_{2}} \otimes \mathbf{1}_{k_{3}}, \quad \boldsymbol{D}^{\prime}{ }_{4}=\boldsymbol{I}_{b} \otimes \boldsymbol{I}_{k_{1}} \otimes \boldsymbol{I}_{k_{2}} \otimes$ $\boldsymbol{I}_{k_{3}}=\boldsymbol{I}_{n}$, where $\boldsymbol{I}_{x}$ is the identity matrix of order $x, \mathbf{1}_{x}$ is the $x$-dimensional vector of ones, and $\otimes$ denotes the Kronecker product of matrices. The $\boldsymbol{\eta}_{f}(f=1,2,3,4)$ are, respectively, random effect vectors of the blocks, the whole plots, the subplots, the sub-subplots, with $E\left(\boldsymbol{\eta}_{f}\right)=\mathbf{0}, \quad$ and $\operatorname{Cov}\left(\boldsymbol{\eta}_{f}\right)=\boldsymbol{V}_{f}$, $\operatorname{Cov}\left(\boldsymbol{\eta}_{f}, \boldsymbol{\eta}_{f^{\prime}}\right)=\mathbf{0}$ for all $f \neq f^{\prime}$. Also $\boldsymbol{e}$ is an $n \times 1$ random vector of technical errors with $E(\boldsymbol{e})=\mathbf{0}$ and $\operatorname{Cov}(\boldsymbol{e})=\sigma_{e}^{2} \boldsymbol{I}_{n}$. According to the orthogonal block 
structure of the considered designs, the covariance matrix $\operatorname{Cov}(\boldsymbol{y})$ can be expressed by

$$
\operatorname{Cov}(\boldsymbol{y})=\gamma_{0} \boldsymbol{P}_{0}+\gamma_{1} \boldsymbol{P}_{1}+\gamma_{2} \boldsymbol{P}_{2}+\gamma_{3} \boldsymbol{P}_{3}+\gamma_{4} \boldsymbol{P}_{4}
$$

where $\gamma_{f} \geq 0$ and $\boldsymbol{P}_{f}$ is a family of known pairwise orthogonal matrices summing to the identity matrix (cf. Mejza, 1997b). The range space of $\boldsymbol{P}_{f}$ for $f=0,1,2,3$, 4 is termed the $f$-th stratum of the model, and $\gamma_{f}$ are unknown stratum variances (cf. Houtman and Speed, 1983).

In the incomplete SSPD the matrices $\boldsymbol{P}_{f}$ represent, respectively, five strata: the total area stratum (zero stratum), the inter-block stratum (the first stratum, I), the inter-whole plot stratum (the second stratum, II), the inter-subplot stratum (the third stratum, III) and the inter-sub-subplot stratum (the fourth stratum, IV).

\section{Construction of an incomplete SSPD}

The incomplete SSPD is characterized by the following incidence matrices: the $v \times b$ incidence matrix $\boldsymbol{N}_{1}=\boldsymbol{\Delta} \boldsymbol{D}^{\prime}{ }_{1}$ (with respect to the blocks), the $v \times b k_{1}$ incidence matrix $\boldsymbol{N}_{2}=\boldsymbol{\Delta} \boldsymbol{D}^{\prime}{ }_{2}$ (with respect to the whole plots), and the $v \times b k_{1} k_{2}$ incidence matrix $\boldsymbol{N}_{3}=\boldsymbol{\Delta} \boldsymbol{D}^{\prime}$ ( (with respect to the subplots). In the design of an experiment the incidence matrix $\boldsymbol{N}_{1}$ plays the most important role. Its form and properties derive uniquely from the adopted method of construction. The remaining incidence matrices follow from the matrix $N_{1}$, but their general forms are not unique. However, the concurrence matrices $\boldsymbol{N}_{i} \boldsymbol{N}^{\prime}{ }_{i}, i=1,2,3$, are unique.

In the method of constructing the incomplete SSPD we need resolvable designs (e.g. Mejza et al., 2012). A design with $v$ treatments is denoted by $\mathrm{D}(v, r, k)$ if each treatment is replicated $r$ times and each block contains $k$ treatments. If the collection of blocks of a $\mathrm{D}(v, r, k)$ can be grouped in such a way that every treatment occurs precisely once in every group, then the design is said to be resolvable and is denoted by $\operatorname{RD}(v, r, k)$. Thus, each group is constituted of one replication of all treatments. $\operatorname{An} \operatorname{RD}(v, r, k)$ such that $v=s^{2}$, $r \leq s+1$ and $k=s$ for a positive integer $s$ is called a square lattice design if any two blocks from different groups contain exactly one common treatment, and 
it is then denoted by $\operatorname{SLD}\left(s^{2}, r, s\right)$. If $r=s+1$, it is called a balanced square lattice design. It is well known that there exists a balanced square lattice design if $s$ is a prime or a prime power (see Raghavarao, 1971).

Let $\boldsymbol{N}_{A}, \boldsymbol{N}_{B}, \boldsymbol{N}_{C}$ be incidence matrices of so-called generating subdesigns for the factors $A, B$ and $C$, respectively. In this construction

$$
N_{A}=\left[N_{A 1}: N_{A 2}: \cdots: N_{A r}\right]
$$

is the incidence matrix of a resolvable design $\operatorname{RD}\left(v^{\prime}, r, k_{1}\right)$, where the $v^{\prime} \times m$ matrix $\boldsymbol{N}_{A i}$ corresponds to the $i$-th replicate, $v^{\prime}\left(=v_{1}\right)$ denotes the number of $A$ treatments, $r$ is the number of their replicates, $k_{1}$ is the number of whole plots inside each block in the SSPD, and $m=\frac{v^{\prime}}{k_{1}}$. Then let

$$
\boldsymbol{N}_{B}=\left[\boldsymbol{N}_{B 1}: \boldsymbol{N}_{B 2}: \cdots: \boldsymbol{N}_{B r}\right] \text { and } \boldsymbol{N}_{C}=\left[\boldsymbol{N}_{C 1}: \boldsymbol{N}_{C 2}: \cdots: \boldsymbol{N}_{C r}\right]
$$

be the incidence matrices of two square lattice designs $\operatorname{SLD}\left(s_{1}^{2}, r, s_{1}\right)$ and $\operatorname{SLD}\left(s_{2}^{2}, r, s_{2}\right)$ with the same number of replicates $r$, where $\boldsymbol{N}_{B i}$ and $\boldsymbol{N}_{C i}$ correspond to the $i$-th replicates with $s_{1}\left(=k_{2}\right)$ and $s_{2}\left(=k_{3}\right)$ plots per block in the SLDs (i.e. the subplots inside each whole plot and the sub-subplots inside each subplot in the SSPD, respectively), and $s_{1}^{2}=v_{2}$ and $s_{2}^{2}=v_{3}$ denote the numbers of $B$ treatments and $C$ treatments respectively.

For the resolvable design $\mathrm{RD}\left(v^{\prime}, r, k_{1}\right)$,

$$
\boldsymbol{N}_{A i}^{\prime} \boldsymbol{N}_{A i}=k_{1} \boldsymbol{I}_{m}
$$

holds for $i=1,2, \ldots, r$. From (3.3), $\boldsymbol{N}_{A i} \boldsymbol{N}_{A i}^{\prime}$ has the eigenvalues $k_{1}$ and 0 with multiplicities $m$ and $v^{\prime}-m$ for each $i=1,2, \ldots, r$. Let $\boldsymbol{P}^{(m)}=$ $\left(\boldsymbol{p}_{0}^{(m)}, \boldsymbol{p}_{1}^{(m)}, \ldots, \boldsymbol{p}_{m-1}^{(m)}\right)$ be an orthogonal matrix of order $m$ with $\boldsymbol{p}_{0}^{(m)}=$ $\left(\boldsymbol{m}^{-1 / 2}\right) \mathbf{1}_{m}$. The mutually orthonormal eigenvectors of $\boldsymbol{N}_{A i} \boldsymbol{N}_{A i}^{\prime}$ corresponding to the eigenvalue $k_{1}$ are given by

$$
\boldsymbol{x}_{i j}=\frac{1}{\sqrt{k_{1}}} \boldsymbol{N}_{A i} \boldsymbol{p}_{j}^{(m)}
$$

for $j=0,1, \ldots, m-1$. In particular $\boldsymbol{x}_{i 0}=\left(\boldsymbol{v}^{\prime}\right)^{-\frac{1}{2}} \mathbf{1}_{\boldsymbol{v}^{\prime}}$. The eigenvectors of $\boldsymbol{N}_{A i} \boldsymbol{N}_{A i}^{\prime}$ corresponding to the eigenvalue 0 are denoted by $\boldsymbol{x}_{i j}^{*}$ for $j=1$, $2, \ldots,\left(v^{\prime}-m\right)$. Here $\boldsymbol{x}_{i 0}, \boldsymbol{x}_{i 1}, \ldots, \boldsymbol{x}_{i m-1}, \boldsymbol{x}_{i 1}^{*}, \boldsymbol{x}_{i 2}^{*}, \ldots, \boldsymbol{x}_{i v^{\prime}-m}^{*}$ are also mutually 
orthonormal. The eigenvalues and the corresponding mutually orthonormal eigenvectors of $\boldsymbol{N}_{A} \boldsymbol{N}_{A}^{\prime}$ are denoted by $\theta_{j}$ and $\boldsymbol{h}_{j}$ for $j=0,1, \ldots, v^{\prime}-1$, with $\theta_{0}=r k_{1}$ and $\boldsymbol{h}_{0}=\frac{1}{\sqrt{v^{\prime}}} \mathbf{1}_{v^{\prime}}$.

For the square lattice designs $\operatorname{SLD}\left(s_{1}^{2}, r, s_{1}\right)$ and $\operatorname{SLD}\left(s_{2}^{2}, r, s_{2}\right)$,

$$
\begin{aligned}
& \boldsymbol{N}_{B i}^{\prime} \boldsymbol{N}_{B i}=s_{1} \boldsymbol{I}_{S_{1}}, \boldsymbol{N}_{B i}^{\prime} \boldsymbol{N}_{B j}=\boldsymbol{J}_{S_{1}}, \\
& \boldsymbol{N}_{C i}^{\prime} \boldsymbol{N}_{C i}=s_{2} \boldsymbol{I}_{S_{2}}, \boldsymbol{N}_{C i}^{\prime} \boldsymbol{N}_{C j}=J_{s_{2}}, \\
& \boldsymbol{N}_{B i} \boldsymbol{N}_{B i}^{\prime} \boldsymbol{N}_{B j} \boldsymbol{N}_{B j}^{\prime}=\boldsymbol{J}_{S_{1}^{2}}, \\
& \boldsymbol{N}_{C i} \boldsymbol{N}_{C i}^{\prime} \boldsymbol{N}_{C j} \boldsymbol{N}_{C j}^{\prime}=\boldsymbol{J}_{S_{2}^{2}},
\end{aligned}
$$

hold for $i, j=1,2, \ldots, r, i \neq j$, where $\boldsymbol{J}_{x}=\mathbf{1}_{x} \mathbf{1}_{x}^{\prime}$. From (3.4), $\boldsymbol{N}_{B i} \boldsymbol{N}_{B i}^{\prime}$ has the eigenvalues $s_{1}$ and 0 with multiplicities $s_{1}$ and $s_{1}\left(s_{1}-1\right)$ for each $i=1,2, \ldots, r$. From (3.6), $\boldsymbol{N}_{B 1} \boldsymbol{N}_{B 1}^{\prime}, \boldsymbol{N}_{B 2} \boldsymbol{N}_{B 2}^{\prime}, \ldots, \boldsymbol{N}_{B r} \boldsymbol{N}_{B r}^{\prime}$ are mutually commutative, so these concurrence matrices have common eigenvectors. For each i-th replicate, from (3.4), the mutually orthonormal eigenvectors of $\boldsymbol{N}_{B i} \boldsymbol{N}_{B i}^{\prime}$ corresponding to the eigenvalue $s_{1}$ are given by $\boldsymbol{z}_{i j}^{B}=\frac{1}{\sqrt{s_{1}}} \boldsymbol{N}_{B i} \boldsymbol{p}_{j}^{\left(s_{1}\right)}$ for $j=0,1, \ldots, s_{1}-1$, where $\boldsymbol{p}_{j}^{\left(s_{1}\right)}$ is the $\mathrm{j}$-th column vector of the orthogonal matrix $\boldsymbol{P}^{\left(s_{1}\right)}\left(\boldsymbol{P}^{\left(s_{1}\right)}=\right.$ $\left.\left(\boldsymbol{p}_{0}^{\left(s_{1}\right)}, \boldsymbol{p}_{1}^{\left(s_{1}\right)}, \ldots, \boldsymbol{p}_{s_{1}-1}^{\left(s_{1}\right)}\right)\right)$. In particular $\boldsymbol{z}_{i 0}^{B}=\frac{1}{s_{1}} \mathbf{1}_{s_{1}^{2}}$. From (3.4), the eigenvectors $\boldsymbol{z}_{i j}^{B}$ for $j=0,1, \ldots, s_{1}-1$ are also the eigenvectors of the concurrence matrix $\boldsymbol{N}_{B h} \boldsymbol{N}_{B h}^{\prime}(h \neq i)$ for the other replicate, and the eigenvalues of $\boldsymbol{N}_{B h} \boldsymbol{N}_{B h}^{\prime}$ corresponding to $\boldsymbol{z}_{i 0}^{B}$ and $\boldsymbol{z}_{i j}^{B}(j \neq 0)$ are $s_{1}$ and 0 respectively. The mutually orthonormal common eigenvectors of $\boldsymbol{N}_{B 1} \boldsymbol{N}_{B 1}^{\prime}, \quad \boldsymbol{N}_{B 2} \boldsymbol{N}_{B 2}^{\prime}, \ldots, \boldsymbol{N}_{B r} \boldsymbol{N}_{B r}^{\prime}$ corresponding to the eigenvalue 0 are denoted by $\boldsymbol{z}_{j}^{* B}$ for $j=1,2, \ldots, s_{1}^{2}-$ $r\left(s_{1}-1\right)-1$. The facts given are summarized in Table 1 .

Here the common eigenvectors in Table 1 are mutually orthogonal. Similarly, from (3.5) and (3.7) we have the eigenvalues and the common eigenvectors of the concurrence matrices $\boldsymbol{N}_{C 1} \boldsymbol{N}_{C 1}^{\prime}, \boldsymbol{N}_{C 2} \boldsymbol{N}_{C 2}^{\prime}, \ldots, \boldsymbol{N}_{C r} \boldsymbol{N}_{C r}^{\prime}$ in $\operatorname{SLD}\left(s_{2}^{2}, r, s_{2}\right)$, which are presented in Table 2.

In the SSPD considered, we can express the incidence matrix $\boldsymbol{N}_{1}$ as

$$
\begin{aligned}
& \boldsymbol{N}_{1}=\boldsymbol{N}_{A} \widetilde{\otimes} \boldsymbol{N}_{B} \widetilde{\otimes} \boldsymbol{N}_{C}=\left[\boldsymbol{N}_{A 1} \otimes \boldsymbol{N}_{B 1} \otimes \boldsymbol{N}_{C 1}: \boldsymbol{N}_{A 2} \otimes \boldsymbol{N}_{B 2} \otimes \boldsymbol{N}_{C 2}:\right. \\
& \left.\cdots: \boldsymbol{N}_{A r} \otimes \boldsymbol{N}_{B r} \otimes \boldsymbol{N}_{C r}\right],
\end{aligned}
$$


Table 1. Eigenvalues and common eigenvectors in $\operatorname{SLD}\left(\boldsymbol{s}_{1}^{2}, \boldsymbol{r}, \boldsymbol{s}_{\mathbf{1}}\right)$

\begin{tabular}{|c|c|c|c|c|}
\hline \multicolumn{4}{|c|}{ Eigenvalues } & \multirow{2}{*}{ Common eigenvectors } \\
\hline $\boldsymbol{N}_{B 1} \boldsymbol{N}_{B 1}^{\prime}$ & $\boldsymbol{N}_{B 2} \boldsymbol{N}_{B 2}^{\prime}$ & $\cdots$ & $\boldsymbol{N}_{B r} \boldsymbol{N}_{B r}^{\prime}$ & \\
\hline$s_{1}$ & $s_{1}$ & $\cdots$ & $s_{1}$ & $\boldsymbol{s}_{1}^{-1} \mathbf{1}_{s_{1}^{2}}$ \\
\hline$s_{1}$ & 0 & $\cdots$ & 0 & $\mathbf{z}_{1 j}^{B}\left(j=1,2, \ldots, s_{1}-1\right)$ \\
\hline 0 & $s_{1}$ & $\cdots$ & 0 & $\mathbf{z}_{2 j}^{B}\left(j=1,2, \ldots, s_{1}-1\right)$ \\
\hline$\vdots$ & $\vdots$ & $\vdots$ & $\vdots$ & $\vdots$ \\
\hline 0 & 0 & $\cdots$ & $s_{1}$ & $\mathbf{z}_{r j}^{B}\left(j=1,2, \ldots, s_{1}-1\right)$ \\
\hline 0 & 0 & $\ldots$ & 0 & $z_{j}^{* B}\left(j=1,2, \ldots, s_{1}^{2}-r\left(s_{1}-1\right)-1\right)$ \\
\hline
\end{tabular}

Table 2. Eigenvalues and common eigenvectors in $\operatorname{SLD}\left(\boldsymbol{s}_{2}^{2}, \boldsymbol{r}, \boldsymbol{s}_{2}\right)$

\begin{tabular}{|c|c|c|c|c|}
\hline \multicolumn{4}{|c|}{ Eigenvalues } & \multirow{2}{*}{ Common eigenvectors } \\
\hline $\boldsymbol{N}_{C 1} \boldsymbol{N}_{C 1}^{\prime}$ & $\boldsymbol{N}_{C 2} \boldsymbol{N}_{C 2}^{\prime}$ & $\ldots$ & $\boldsymbol{N}_{C r} \boldsymbol{N}_{c}^{\prime}$ & \\
\hline$s_{2}$ & $s_{2}$ & $\cdots$ & $s_{2}$ & $\boldsymbol{s}_{2}^{-1} \mathbf{1}_{s_{2}^{2}}$ \\
\hline$s_{2}$ & 0 & $\cdots$ & 0 & $z_{1 j}^{C}\left(j=1,2, \ldots, s_{2}-1\right)$ \\
\hline 0 & $s_{2}$ & $\cdots$ & 0 & $\mathbf{z}_{2 j}^{C}\left(j=1,2, \ldots, s_{2}-1\right)$ \\
\hline$\vdots$ & $\vdots$ & $\vdots$ & $\vdots$ & $\vdots$ \\
\hline 0 & 0 & $\cdots$ & $s_{2}$ & $\mathbf{z}_{r j}^{C}\left(j=1,2, \ldots, s_{2}-1\right)$ \\
\hline 0 & 0 & $\ldots$ & 0 & $\boldsymbol{z}_{j}^{* C}\left(j=1,2, \ldots, s_{2}^{2}-r\left(s_{2}-1\right)-1\right)$ \\
\hline
\end{tabular}

which is called the semi-Kronecker product of $\boldsymbol{N}_{A}, \boldsymbol{N}_{B}$ and $\boldsymbol{N}_{C}$. From (3.8), the concurrence matrices have the forms:

$$
\begin{aligned}
& \boldsymbol{N}_{1} \boldsymbol{N}_{1}^{\prime}=\sum_{i=1}^{r}\left(\left(\boldsymbol{N}_{A i} \boldsymbol{N}_{A i}^{\prime}\right) \otimes\left(\boldsymbol{N}_{B i} \boldsymbol{N}_{B i}^{\prime}\right) \otimes\left(\boldsymbol{N}_{C i} \boldsymbol{N}_{C i}^{\prime}\right)\right), \\
& \boldsymbol{N}_{2} \boldsymbol{N}_{2}^{\prime}=\sum_{i=1}^{r}\left(\boldsymbol{I}_{v^{\prime}} \otimes\left(\boldsymbol{N}_{B i} \boldsymbol{N}_{B i}^{\prime}\right) \otimes\left(\boldsymbol{N}_{C i} \boldsymbol{N}_{C i}^{\prime}\right)\right), \\
& \boldsymbol{N}_{3} \boldsymbol{N}_{3}^{\prime}=\sum_{i=1}^{r}\left(\boldsymbol{I}_{v^{\prime}} \otimes \boldsymbol{I}_{S_{1}^{2}} \otimes\left(\boldsymbol{N}_{C i} \boldsymbol{N}_{C i}^{\prime}\right)\right) .
\end{aligned}
$$

In this method of construction the number of treatment combinations is equal to $v=v_{1} v_{2} v_{3}=v^{\prime} s_{1}^{2} s_{2}^{2}$, and the number of all units is $n=b k_{1} k_{2} k_{3}=b k_{1} s_{1} s_{2}$. 
This means that the incomplete SSPD has $v^{\prime}$ whole plot $(A)$ treatments, $s_{1}^{2}$ subplot $(B)$ treatments and $s_{2}^{2}$ sub-subplot $(C)$ treatments.

\section{Analysis}

The statistical analysis of submodels related to the different strata (2.3) is based on algebraic properties of stratum information matrices for the treatment combinations, which are defined as

$$
\boldsymbol{A}_{f}=\Delta \boldsymbol{P}_{\boldsymbol{f}} \boldsymbol{\Delta}^{\prime}
$$

for $f=0,1,2,3,4$. In the SSPD considered, from (4.1) we have

$$
\begin{aligned}
& \boldsymbol{A}_{0}=\frac{r}{v} \boldsymbol{J}_{v}, \quad \boldsymbol{A}_{1}=\frac{1}{k_{1} s_{1} s_{2}} \boldsymbol{N}_{1} \boldsymbol{N}^{\prime}{ }_{1}-\frac{r}{v} \boldsymbol{J}_{v}, \quad \boldsymbol{A}_{2}=\frac{1}{s_{1} s_{2}} \boldsymbol{N}_{2} \boldsymbol{N}^{\prime}{ }_{2}-\frac{1}{k_{1} s_{1} s_{2}} \boldsymbol{N}_{1} \boldsymbol{N}^{\prime}{ }_{1}, \\
& \boldsymbol{A}_{3}=\frac{1}{s_{2}} \boldsymbol{N}_{3} \boldsymbol{N}^{\prime}{ }_{3}-\frac{1}{s_{1} s_{2}} \boldsymbol{N}_{2} \boldsymbol{N}^{\prime}{ }_{2}, \quad \boldsymbol{A}_{4}=r \boldsymbol{I}_{v}-\frac{1}{s_{2}} \boldsymbol{N}_{3} \boldsymbol{N}^{\prime}{ }_{3},
\end{aligned}
$$

where $v=v^{\prime} s_{1}^{2} s_{2}^{2}$.

Our considerations concern an incomplete SSPD with the general balance (GB) property only, i.e. the matrices $\boldsymbol{A}_{f}(f=0,1,2,3,4)$ mutually commute. From this fact let us note that the following relations hold:

$$
\begin{aligned}
& N_{1} N_{1}^{\prime} N_{2} N^{\prime}{ }_{2}=N_{2} N_{2}{ }_{2} N_{1} N_{1}^{\prime}, \\
& N_{1} N_{1}^{\prime} N_{3} N_{3}^{\prime}=N_{3} N_{3}^{\prime} N_{1} N_{1}^{\prime}, \\
& N_{2} N_{2}^{\prime} N_{3} N_{3}^{\prime}=N_{3} N_{3}^{\prime} N_{2} N^{\prime}{ }_{2} .
\end{aligned}
$$

This means that the matrices (4.2), and therefore (4.3), have a common set of eigenvectors $\left\{\boldsymbol{p}_{h}\right\}$ corresponding to eigenvalues $\left\{\varepsilon_{f h}\right\}$ with respect to $r$, where $0 \leq \varepsilon_{f h} \leq 1$ and $f=0,1,2,3,4 ; h=1,2, \ldots, v$. Since $\boldsymbol{A}_{f} \mathbf{1}_{v}=\mathbf{0}$ for $f>0$, for instance, the last eigenvector may be chosen as $\boldsymbol{p}_{v}=n^{-1 / 2} \mathbf{1}_{v}$. The remaining eigenvectors $\left\{\boldsymbol{p}_{h}\right\}$ for $h<v$, where $\boldsymbol{p}_{h}^{\prime} \boldsymbol{p}_{v}=0$, form a basis for all vectors generating some contrasts. Also, from the relation $\boldsymbol{A}_{0} \mathbf{1}_{v} \neq \mathbf{0}$, it follows that the zero stratum (for $f=0$ ) is connected with estimation of the general mean only.

It can be easily shown that the mutually orthonormal common eigenvectors of $\boldsymbol{N}_{1} \boldsymbol{N}_{1}{ }_{1}, \boldsymbol{N}_{2} \boldsymbol{N}^{\prime}{ }_{2}$ and $\boldsymbol{N}_{3} \boldsymbol{N}^{\prime}{ }_{3}$ are as follows: 
(1) $\quad \frac{1}{\sqrt{v^{\prime}}} \mathbf{1}_{v^{\prime}} \otimes \frac{1}{s_{1}} \mathbf{1}_{s_{1}^{2}} \otimes \frac{1}{s_{2}} \mathbf{1}_{s_{2}^{2}}$,

(2) $\quad \boldsymbol{h}_{j} \otimes \frac{1}{s_{1}} \mathbf{1}_{s_{1}^{2}} \otimes \frac{1}{s_{2}} \mathbf{1}_{s_{2}^{2}},\left(j=1,2, \ldots, v^{\prime}-1\right)$,

(3) $\frac{1}{\sqrt{v^{\prime}}} \mathbf{1}_{v^{\prime}} \otimes \frac{1}{s_{1}} \mathbf{1}_{s_{1}^{2}} \otimes \mathbf{z}_{i j}^{C},\left(i=1,2, \ldots, r, j=1,2, \ldots, s_{2}-1\right)$,

(4) $\quad \boldsymbol{x}_{i j} \otimes \frac{1}{s_{1}} \mathbf{1}_{s_{1}^{2}} \otimes \mathbf{z}_{i j^{\prime}}^{C},\left(i=1,2, \ldots, r, j=1,2, \ldots, m-1, j^{\prime}=1,2, \ldots, s_{2}-1\right)$,

(5) $\quad \boldsymbol{x}_{i j}^{*} \otimes \frac{1}{s_{1}} \mathbf{1}_{s_{1}^{2}} \otimes \mathbf{z}_{i j^{\prime}}^{C},\left(i=1,2, \ldots, r, j=1,2, \ldots, v^{\prime}-m, j^{\prime}=1,2, \ldots, s_{2}-1\right)$,

(6) $\quad \frac{1}{\sqrt{v^{\prime}}} \mathbf{1}_{v^{\prime}} \otimes \frac{1}{s_{1}} \mathbf{1}_{s_{1}^{2}} \otimes \mathbf{z}_{j}^{* C},\left(j=1,2, \ldots, s_{2}^{2}-r\left(s_{2}-1\right)-1\right)$,

(7) $\quad \boldsymbol{h}_{j} \otimes \frac{1}{s_{1}} \mathbf{1}_{s_{1}^{2}} \otimes \mathbf{z}_{j^{\prime}}^{* C},\left(j=1,2, \ldots, v^{\prime}-1, j^{\prime}=1,2, \ldots, s_{2}^{2}-r\left(s_{2}-1\right)-1\right)$,

(8) $\quad \frac{1}{\sqrt{v^{\prime}}} \mathbf{1}_{v^{\prime}} \otimes \mathbf{z}_{i j}^{B} \otimes \frac{1}{s_{2}} \mathbf{1}_{s_{2}^{2}},\left(i=1,2, \ldots, r, j=1,2, \ldots, s_{1}-1\right)$,

(9) $\quad \boldsymbol{x}_{i j} \otimes \mathbf{z}_{i j^{\prime}}^{B} \otimes \frac{1}{s_{2}} \mathbf{1}_{s_{2}^{2}},\left(i=1,2, \ldots, r, j=1,2, \ldots, m-1, j^{\prime}=1,2, \ldots, s_{1}-1\right)$,

(10) $\boldsymbol{x}_{i j}^{*} \otimes \mathbf{z}_{i j^{\prime}}^{B} \otimes \frac{1}{s_{2}} \mathbf{1}_{s_{2}^{2}},\left(i=1,2, \ldots, r, j=1,2, \ldots, v^{\prime}-m, j^{\prime}=1,2, \ldots, s_{1}-1\right)$,

(11) $\frac{1}{\sqrt{v^{\prime}}} \mathbf{1}_{v^{\prime}} \otimes \mathbf{z}_{i j}^{B} \otimes \mathbf{z}_{i j^{\prime}}^{C},\left(i=1,2, \ldots, r, j=1,2, \ldots, s_{1}-1, j^{\prime}=1,2, \ldots, s_{2}-1\right)$,

(12) $\quad \boldsymbol{x}_{i j} \otimes \mathbf{z}_{i j^{\prime}}^{B} \otimes \mathbf{z}_{i j^{\prime \prime}}^{C}, \quad\left(i=1,2, \ldots, r, j=1,2, \ldots, m-1, j^{\prime}=1,2, \ldots, s_{1}-1, j^{\prime \prime}=\right.$ $\left.1,2, \ldots, s_{2}-1\right)$,

(13) $\quad \boldsymbol{x}_{i j}^{*} \otimes \mathbf{z}_{i j^{\prime}}^{B} \otimes \mathbf{z}_{i j^{\prime \prime}}^{C}, \quad\left(i=1,2, \ldots, r, j=1,2, \ldots, v^{\prime}-m, j^{\prime}=1,2, \ldots, s_{1}-1, j^{\prime \prime}=\right.$ $\left.1,2, \ldots, s_{2}-1\right)$,

(14) $\frac{1}{\sqrt{v^{\prime}}} \mathbf{1}_{v^{\prime}} \otimes \mathbf{z}_{i j}^{B} \otimes \mathbf{z}_{i^{\prime} j^{\prime}}^{C},\left(i \neq i^{\prime}, i, i^{\prime}=1,2, \ldots, r, j=1,2, \ldots, s_{1}-1, j^{\prime}=1,2, \ldots, s_{2}-1\right)$,

(15) $\quad \boldsymbol{h}_{j} \otimes \mathbf{z}_{i j^{\prime}}^{B} \otimes \mathbf{z}_{i^{\prime} j^{\prime \prime}}^{C},\left(j=1,2, \ldots, v^{\prime}-1, i \neq i^{\prime}, i, i^{\prime}=1,2, \ldots, r, j^{\prime}=1,2, \ldots, s_{1}-1\right.$, $\left.j^{\prime \prime}=1,2, \ldots, s_{2}-1\right)$,

(16) $\frac{1}{\sqrt{v^{\prime}}} \mathbf{1}_{v^{\prime}} \otimes \mathbf{z}_{i j}^{B} \otimes \mathbf{z}_{j^{\prime}}^{* C},\left(i=1,2, \ldots, r, j=1,2, \ldots, s_{1}-1, j^{\prime}=1, \ldots, s_{2}^{2}-r\left(s_{2}-1\right)-1\right)$,

(17) $\quad \boldsymbol{h}_{j} \otimes \mathbf{z}_{i j^{\prime}}^{B} \otimes \mathbf{z}_{j^{\prime \prime}}^{* C},\left(j=1,2, \ldots, v^{\prime}-1, i=1,2, \ldots, r, j^{\prime}=1,2, \ldots, s_{1}-1, j^{\prime \prime}=\right.$ $\left.1,2, \ldots, s_{2}^{2}-r\left(s_{2}-1\right)-1\right)$,

(18) $\frac{1}{\sqrt{v^{\prime}}} \mathbf{1}_{v^{\prime}} \otimes \mathbf{z}_{j}^{* B} \otimes \frac{1}{s_{2}} \mathbf{1}_{s_{2}^{2}},\left(j=1,2, \ldots, s_{1}^{2}-r\left(s_{1}-1\right)-1\right)$,

(19) $\boldsymbol{h}_{j} \otimes \mathbf{z}_{j^{\prime}}^{* B} \otimes \frac{1}{s_{2}} \mathbf{1}_{s_{2}^{2}},\left(j=1,2, \ldots, v^{\prime}-1, j^{\prime}=1,2, \ldots, s_{1}^{2}-r\left(s_{1}-1\right)-1\right)$, 
(20) $\frac{1}{\sqrt{v^{\prime}}} \mathbf{1}_{v^{\prime}} \otimes \mathbf{z}_{j}^{* B} \otimes \mathbf{z}_{i j^{\prime}}^{C}, \quad\left(j=1, \ldots, s_{1}^{2}-r\left(s_{1}-1\right)-1, i=1,2, \ldots, r, j^{\prime}=1, \ldots, s_{2}-1\right)$,

(21) $\boldsymbol{h}_{j} \otimes \mathbf{z}_{j^{\prime}}^{* B} \otimes \mathbf{z}_{i j^{\prime \prime}}^{C},\left(j=1,2, \ldots, v^{\prime}-1, j^{\prime}=1,2, \ldots, s_{1}^{2}-r\left(s_{1}-1\right)-1, i=\right.$ $\left.1,2, \ldots, r, j^{\prime \prime}=1,2, \ldots, s_{2}-1\right)$,

(22) $\frac{1}{\sqrt{v^{\prime}}} \mathbf{1}_{v^{\prime}} \otimes \mathbf{z}_{j}^{* B} \otimes \mathbf{z}_{j^{\prime}}^{* C}, \quad\left(j=1,2, \ldots, s_{1}^{2}-r\left(s_{1}-1\right)-1, j^{\prime}=1,2, \ldots, s_{2}^{2}-\right.$ $\left.r\left(s_{2}-1\right)-1\right)$,

(23) $\boldsymbol{h}_{j} \otimes \mathbf{z}_{j^{\prime}}^{* B} \otimes \mathbf{z}_{j^{\prime \prime}}^{* C},\left(j=1,2, \ldots, v^{\prime}-1, j^{\prime}=1,2, \ldots, s_{1}^{2}-r\left(s_{1}-1\right)-1, j^{\prime \prime}=\right.$ $\left.1,2, \ldots, s_{2}^{2}-r\left(s_{2}-1\right)-1\right)$.

In Table 3 we present eigenvalues and corresponding common eigenvectors (1)-(23) of the concurrence matrices $\boldsymbol{N}_{i} \boldsymbol{N}_{i}^{\prime}, i=1,2,3$.

Table 3. Eigenvalues and common eigenvectors of $N_{1} N_{1}^{\prime}, N_{2} N_{2}^{\prime}, N_{3} N_{3}^{\prime}$

\begin{tabular}{cccc}
\hline \multicolumn{2}{c}{ Eigenvalues } & & Common eigenvectors \\
\hline $\boldsymbol{N}_{1} \boldsymbol{N}_{1}^{\prime}$ & $\boldsymbol{N}_{2} \boldsymbol{N}_{2}^{\prime}$ & $\boldsymbol{N}_{3} \boldsymbol{N}_{3}^{\prime}$ & \\
$r k_{1} s_{1} s_{2}$ & $r s_{1} s_{2}$ & $r s_{2}$ & $(1)$ \\
$s_{1} s_{2} \theta_{j} j=1,2, \ldots, v^{\prime}-1$ & $r s_{1} s_{2}$ & $r s_{2}$ & $(3),(4),(11),(12)$ \\
$k_{1} s_{1} s_{2}$ & $s_{1} s_{2}$ & $s_{2}$ & $(5),(13)$ \\
0 & $s_{1} s_{2}$ & $s_{2}$ & $(6),(7),(16),(17),(22),(23)$ \\
0 & 0 & 0 & $(8),(9)$ \\
$k_{1} s_{1} s_{2}$ & $s_{1} s_{2}$ & $r s_{2}$ & $(10)$ \\
0 & $s_{1} s_{2}$ & $r s_{2}$ & $(14),(15),(20),(21)$ \\
0 & 0 & $s_{2}$ & $(18),(19)$ \\
0 & 0 & $r s_{2}$ &
\end{tabular}

We can note that any vector $\boldsymbol{c}_{h}=r \boldsymbol{p}_{h}$ such that the eigenvector $\boldsymbol{p}_{h}$ satisfies the condition

$$
\boldsymbol{A}_{f} \boldsymbol{p}_{h}=r \varepsilon_{f h} \boldsymbol{p}_{h}
$$

for $f=1,2,3,4 ; h=1,2, \ldots, v-1$ defines an orthogonal (basic) contrast $\boldsymbol{c}^{\prime}{ }_{h} \boldsymbol{\tau}$ (cf. Pearce et al., 1974). The $\varepsilon_{f h}$ is called the stratum efficiency factor for the 
SSPD. The contrasts are connected with comparisons among main effects of the considered factors and interaction effects between them. They can be expressed through the eigenvectors given in Table 3. The vectors (1)-(23) are also the common eigenvectors of the stratum information matrices (4.2) with respect to $r$.

Finally we present potential stratum efficiency factors for the incomplete SSPD.

Table 4. Stratum efficiency factors for the incomplete SSPD

\begin{tabular}{|c|c|c|c|c|c|}
\hline \multirow{2}{*}{\multicolumn{2}{|c|}{$\begin{array}{l}\text { Type of contrasts } \\
\text { (No. of vectors) }\end{array}$}} & \multicolumn{4}{|c|}{ Strata } \\
\hline & & \multirow{2}{*}{$\frac{\mathrm{I}}{\theta_{j} /\left(r k_{1}\right)}$} & \multirow{2}{*}{$\frac{\mathrm{II}}{1-\theta_{j} /\left(r k_{1}\right)}$} & \multirow{2}{*}{$\begin{array}{c}\text { III } \\
-\end{array}$} & \multirow{2}{*}{$\begin{array}{l}\text { IV } \\
-\end{array}$} \\
\hline$A$ & (2) & & & & \\
\hline \multirow[t]{2}{*}{$B$} & (8) & $1 / r$ & - & $1-1 / r$ & - \\
\hline & (18) & - & - & 1 & - \\
\hline \multirow[t]{2}{*}{$C$} & (3) & $1 / r$ & - & - & $1-1 / r$ \\
\hline & (6) & - & - & - & 1 \\
\hline \multirow[t]{3}{*}{$A \times B$} & (9) & $1 / r$ & - & $1-1 / r$ & - \\
\hline & (10) & - & $1 / r$ & $1-1 / r$ & - \\
\hline & (19) & - & - & 1 & - \\
\hline \multirow[t]{3}{*}{$A \times C$} & (4) & $1 / r$ & - & - & $1-1 / r$ \\
\hline & (5) & - & $1 / r$ & - & $1-1 / r$ \\
\hline & (7) & - & - & - & 1 \\
\hline \multirow[t]{3}{*}{$B \times C$} & (11) & $1 / r$ & - & - & $1-1 / r$ \\
\hline & (14) (20) & - & - & $1 / r$ & $1-1 / r$ \\
\hline & (16) (22) & - & - & - & 1 \\
\hline \multirow[t]{4}{*}{$A \times B \times C$} & (12) & $1 / r$ & - & - & $1-1 / r$ \\
\hline & (13) & - & $1 / r$ & - & $1-1 / r$ \\
\hline & (15) (21) & - & - & $1 / r$ & $1-1 / r$ \\
\hline & (17) (23) & - & - & - & 1 \\
\hline
\end{tabular}

Here $j=1,2, \ldots, v^{\prime}-1$, and I, II, III and IV denote the inter-block stratum, the inter-whole plot stratum, the inter-subplot stratum and the inter-sub-subplot stratum, respectively. 


\section{Example and discussion}

Consider a $(6 \times 4 \times 9)$-experiment arranged in an incomplete SSPD. The $A$ treatments are allocated in a resolvable design with the incidence matrix $\boldsymbol{N}_{A}=$ $\left(\boldsymbol{N}_{A 1}: \boldsymbol{N}_{A 2}: \boldsymbol{N}_{A 3}\right)(\operatorname{RD}(6,3,3))$ (cf. Mejza et al., 2012). The $\operatorname{RD}(6,3,3)$ is an optimal $\alpha$-design generated by the CycDesigN software (see Whitaker et al., 2002). The parameters are as follows: $v^{\prime}=6, r=k_{1}=3$.

In turn, the $B$ treatments $\left(s_{1}^{2}=4\right)$ and $C$ treatments $\left(s_{2}^{2}=9\right)$ are allocated in different square lattice designs with the incidence matrices $\boldsymbol{N}_{B}=$ $\left(\boldsymbol{N}_{B 1}: \boldsymbol{N}_{B 2}: \boldsymbol{N}_{B 3}\right)(\operatorname{SLD}(4,3,2))$ and $\boldsymbol{N}_{C}=\left(\boldsymbol{N}_{C 1}: \boldsymbol{N}_{C 2}: \boldsymbol{N}_{C 3}\right)(\operatorname{SLD}(9,3,3))$ respectively. Assume that these incidence matrices have the following forms (see Clatworthy, 1973):

$$
\begin{aligned}
& \boldsymbol{N}_{A}=\left[\begin{array}{llllllll}
1 & 0 & \vdots & 1 & 0 & \vdots & 1 & 0 \\
1 & 0 & \vdots & 1 & 0 & \vdots & 0 & 1 \\
1 & 0 & \vdots & 0 & 1 & \vdots & 1 & 0 \\
0 & 1 & \vdots & 0 & 1 & \vdots & 0 & 1 \\
0 & 1 & \vdots & 0 & 1 & \vdots & 1 & 0 \\
0 & 1 & \vdots & 1 & 0 & \vdots & 0 & 1
\end{array}\right], \\
& \boldsymbol{N}_{B}=\left[\begin{array}{cccccccc}
1 & 0 & \vdots & 1 & 0 & \vdots & 1 & 0 \\
1 & 0 & \vdots & 0 & 1 & \vdots & 0 & 1 \\
0 & 1 & \vdots & 1 & 0 & \vdots & 0 & 1 \\
0 & 1 & \vdots & 0 & 1 & \vdots & 1 & 0
\end{array}\right] \text {, } \\
& \boldsymbol{N}_{C}=\left[\begin{array}{lllllllllll}
1 & 0 & 0 & \vdots & 1 & 0 & 0 & \vdots & 1 & 0 & 0 \\
1 & 0 & 0 & \vdots & 0 & 1 & 0 & \vdots & 0 & 1 & 0 \\
1 & 0 & 0 & \vdots & 0 & 0 & 1 & \vdots & 0 & 0 & 1 \\
0 & 1 & 0 & \vdots & 1 & 0 & 0 & \vdots & 0 & 0 & 1 \\
0 & 1 & 0 & \vdots & 0 & 1 & 0 & \vdots & 1 & 0 & 0 \\
0 & 1 & 0 & \vdots & 0 & 0 & 1 & \vdots & 0 & 1 & 0 \\
0 & 0 & 1 & \vdots & 1 & 0 & 0 & \vdots & 0 & 1 & 0 \\
0 & 0 & 1 & \vdots & 0 & 1 & 0 & \vdots & 0 & 0 & 1 \\
0 & 0 & 1 & \vdots & 0 & 0 & 1 & \vdots & 1 & 0 & 0
\end{array}\right] \text { with } 3 \text { replicates. }
\end{aligned}
$$

From (3.3), $\boldsymbol{N}_{A i} \boldsymbol{N}_{A i}^{\prime}$ has the eigenvalues $k_{1}=3$ and 0 with multiplicities $m$ $\left(=v^{\prime} k_{1}^{-1}\right)=2$ and $v^{\prime}-m=4$ for each $i=1,2,3$. The eigenvectors of $\boldsymbol{N}_{A i} \boldsymbol{N}_{A i}^{\prime}$ corresponding to the eigenvalue 0 are denoted by $\boldsymbol{x}_{i j}^{*}$ for $j=1,2,3,4$. Furthermore, the eigenvalues and the corresponding eigenvectors of $\boldsymbol{N}_{A} \boldsymbol{N}_{A}^{\prime}$ are 
denoted by $\theta_{j}$ and $\boldsymbol{h}_{j}$ for $j=0,1, \ldots, 5$, with $\theta_{0}=r k_{1}=9$ and $\boldsymbol{h}_{0}=\frac{1}{\sqrt{v^{\prime}}} \mathbf{1}_{v^{\prime}}=$ $\frac{1}{\sqrt{6}} \mathbf{1}_{6}$. From Tables $1-2$ we have that $\boldsymbol{N}_{B i} \boldsymbol{N}_{B i}^{\prime}(\mathrm{i}=1,2,3)$ has the eigenvalues $s_{1}$ $=2$ and 0 , with multiplicities $s_{1}=2$ and $s_{1}\left(s_{1}-1\right)=2$ respectively. In turn, $\boldsymbol{N}_{C i} \boldsymbol{N}_{C i}^{\prime}(\mathrm{i}=1,2,3)$ has the eigenvalues $s_{2}=3$ and 0 , with multiplicities $s_{2}=3$ and $s_{2}\left(s_{2}-1\right)=6$.

Using the semi-Kronecker product $\boldsymbol{N}_{1}=\boldsymbol{N}_{A} \widetilde{\otimes} \boldsymbol{N}_{B} \widetilde{\otimes} \boldsymbol{N}_{C}$ (see (3.8)) we obtain the incomplete SSPD with parameters: $v=v^{\prime} s_{1}^{2} s_{2}^{2}=216, \quad b=$ $r m s_{1} s_{2}=36, k=k_{1} s_{1} s_{2}=18, r=3$ and $n=648$, where $m=\frac{v^{\prime}}{k_{1}}=2, s_{1}=$ $2, s_{2}=3$ and $k_{1}=3$.

Table 5. Eigenvalues and common eigenvectors of $N_{1} N_{1}^{\prime}, N_{2} N_{2}^{\prime}$ and $N_{3} N_{3}^{\prime}$

\begin{tabular}{|c|c|c|c|}
\hline \multicolumn{3}{|c|}{ Eigenvalues } & \multirow{2}{*}{ Common eigenvectors } \\
\hline$N_{1} N_{1}^{\prime}$ & $\mathrm{N}_{2} \mathrm{~N}_{2}^{\prime}$ & $N_{3} N_{3}^{\prime}$ & \\
\hline$r k_{1} s_{1} s_{2}=54$ & $r s_{1} s_{2}=18$ & $r s_{2}=9$ & (1) \\
\hline$s_{1} s_{2} \theta_{j}=6 \cdot \theta_{j} j=1, \ldots, 5$ & $r s_{1} s_{2}=18$ & $r s_{2}=9$ & (2) \\
\hline$k_{1} s_{1} s_{2}=18$ & $s_{1} s_{2}=6$ & $s_{2}=3$ & (3), (4), (11), (12) \\
\hline 0 & $s_{1} s_{2}=6$ & $s_{2}=3$ & $(5),(13)$ \\
\hline 0 & 0 & 0 & (6), (7), (16), (17), (22), (23) \\
\hline$k_{1} s_{1} s_{2}=18$ & $s_{1} s_{2}=6$ & $r s_{2}=9$ & (8), (9) \\
\hline 0 & $s_{1} s_{2}=6$ & $r s_{2}=9$ & (10) \\
\hline 0 & 0 & $s_{2}=3$ & (14), (15), (20), (21) \\
\hline 0 & 0 & $r s_{2}=9$ & $(18),(19)$ \\
\hline
\end{tabular}

Let us note that the vectors (1)-(17) are also the common eigenvectors of the stratum information matrices (4.2) with respect to $r$. The vectors (18)-(23) do not occur in the example considered.

To present a sample layout of the considered SSPD, we introduce an abbreviation. Let $\left\{A_{i}, A_{j}, A_{d}\left|B_{w}, B_{z}\right| C_{l}, C_{m}, C_{p}\right\}$ denote a block such that $A_{i}, A_{j}, A_{d}$, where $i, j, d \in\{1,2,3,4,5,6\}, i \neq j \neq d \neq i$, are the whole plot treatments inside the block, $B_{w}, B_{z}$, where $w, z \in\{1,2,3,4\}, w \neq z$, are the subplot treatments in each of the whole plots, and $C_{l}, C_{m}, C_{p}$, where $l, m, p \in$ 
$\{1,2,3,4,5,6,7,8,9\}, l \neq m \neq p \neq l$, are the sub-subplot treatments in each of the subplots inside the block. The contents of the blocks can be expressed as follows (before randomization of units):

$\left\{A_{1}, A_{2}, A_{3}\left|B_{1}, B_{2}\right| C_{1}, C_{2}, C_{3}\right\},\left\{A_{1}, A_{2}, A_{3}\left|B_{1}, B_{2}\right| C_{4}, C_{5}, C_{6}\right\},\left\{A_{1}, A_{2}, A_{3}\left|B_{1}, B_{2}\right| C_{7}, C_{8}, C_{9}\right\}$, $\left\{A_{1}, A_{2}, A_{3}\left|B_{3}, B_{4}\right| C_{1}, C_{2}, C_{3}\right\},\left\{A_{1}, A_{2}, A_{3}\left|B_{3}, B_{4}\right| C_{4}, C_{5}, C_{6}\right\},\left\{A_{1}, A_{2}, A_{3}\left|B_{3}, B_{4}\right| C_{7}, C_{8}, C_{9}\right\}$, $\left\{A_{4}, A_{5}, A_{6}\left|B_{1}, B_{2}\right| C_{1}, C_{2}, C_{3}\right\},\left\{A_{4}, A_{5}, A_{6}\left|B_{1}, B_{2}\right| C_{4}, C_{5}, C_{6}\right\},\left\{A_{4}, A_{5}, A_{6}\left|B_{1}, B_{2}\right| C_{7}, C_{8}, C_{9}\right\}$, $\left\{A_{4}, A_{5}, A_{6}\left|B_{3}, B_{4}\right| C_{1}, C_{2}, C_{3}\right\},\left\{A_{4}, A_{5}, A_{6}\left|B_{3}, B_{4}\right| C_{4}, C_{5}, C_{6}\right\},\left\{A_{4}, A_{5}, A_{6}\left|B_{3}, B_{4}\right| C_{7}, C_{8}, C_{9}\right\}$, $\left\{A_{1}, A_{2}, A_{6}\left|B_{1}, B_{3}\right| C_{1}, C_{4}, C_{7}\right\},\left\{A_{1}, A_{2}, A_{6}\left|B_{1}, B_{3}\right| C_{2}, C_{5}, C_{8}\right\},\left\{A_{1}, A_{2}, A_{6}\left|B_{1}, B_{3}\right| C_{3}, C_{6}, C_{9}\right\}$, $\left\{A_{1}, A_{2}, A_{6}\left|B_{2}, B_{4}\right| C_{1}, C_{4}, C_{7}\right\},\left\{A_{1}, A_{2}, A_{6}\left|B_{2}, B_{4}\right| C_{2}, C_{5}, C_{8}\right\},\left\{A_{1}, A_{2}, A_{6}\left|B_{2}, B_{4}\right| C_{3}, C_{6}, C_{9}\right\}$, $\left\{A_{3}, A_{4}, A_{5}\left|B_{1}, B_{3}\right| C_{1}, C_{4}, C_{7}\right\},\left\{A_{3}, A_{4}, A_{5}\left|B_{1}, B_{3}\right| C_{2}, C_{5}, C_{8}\right\},\left\{A_{3}, A_{4}, A_{5}\left|B_{1}, B_{3}\right| C_{3}, C_{6}, C_{9}\right\}$, $\left\{A_{3}, A_{4}, A_{5}\left|B_{2}, B_{4}\right| C_{1}, C_{4}, C_{7}\right\},\left\{A_{3}, A_{4}, A_{5}\left|B_{2}, B_{4}\right| C_{2}, C_{5}, C_{8}\right\},\left\{A_{3}, A_{4}, A_{5}\left|B_{2}, B_{4}\right| C_{3}, C_{6}, C_{9}\right\}$, $\left\{A_{1}, A_{3}, A_{5}\left|B_{1}, B_{4}\right| C_{1}, C_{5}, C_{9}\right\},\left\{A_{1}, A_{3}, A_{5}\left|B_{1}, B_{4}\right| C_{2}, C_{6}, C_{7}\right\},\left\{A_{1}, A_{3}, A_{5}\left|B_{1}, B_{4}\right| C_{3}, C_{4}, C_{8}\right\}$, $\left\{A_{1}, A_{3}, A_{5}\left|B_{2}, B_{3}\right| C_{1}, C_{5}, C_{9}\right\},\left\{A_{1}, A_{3}, A_{5}\left|B_{2}, B_{3}\right| C_{2}, C_{6}, C_{7}\right\},\left\{A_{1}, A_{3}, A_{5}\left|B_{2}, B_{3}\right| C_{3}, C_{4}, C_{8}\right\}$, $\left\{A_{2}, A_{4}, A_{6}\left|B_{1}, B_{4}\right| C_{1}, C_{5}, C_{9}\right\},\left\{A_{2}, A_{4}, A_{6}\left|B_{1}, B_{4}\right| C_{2}, C_{6}, C_{7}\right\},\left\{A_{2}, A_{4}, A_{6}\left|B_{1}, B_{4}\right| C_{3}, C_{4}, C_{8}\right\}$, $\left\{A_{2}, A_{4}, A_{6}\left|B_{2}, B_{3}\right| C_{1}, C_{5}, C_{9}\right\},\left\{A_{2}, A_{4}, A_{6}\left|B_{2}, B_{3}\right| C_{2}, C_{6}, C_{7}\right\},\left\{A_{2}, A_{4}, A_{6}\left|B_{2}, B_{3}\right| C_{3}, C_{4}, C_{8}\right\}$.

Below we show an example of a single block from the sample layout (after three-step randomization). Let us consider the last of them, i.e. $\left\{A_{2}, A_{4}, A_{6} \mid B_{2}\right.$, $\left.B_{3} \mid C_{3}, C_{4}, C_{8}\right\}$.

\begin{tabular}{|c|c|c|c|c|c|c|c|c|c|c|c|c|c|c|c|c|c|}
\hline \multicolumn{6}{|c|}{$A_{6}$} & \multicolumn{6}{|c|}{$A_{2}$} & \multicolumn{6}{|c|}{$A_{4}$} \\
\hline \multicolumn{3}{|c|}{$B_{3}$} & \multicolumn{3}{|c|}{$B_{2}$} & \multicolumn{3}{|c|}{$B_{2}$} & \multicolumn{3}{|c|}{$B_{3}$} & \multicolumn{3}{|c|}{$B_{3}$} & \multicolumn{3}{|c|}{$B_{2}$} \\
\hline$C_{4}$ & $C_{8}$ & $C_{3}$ & $C_{8}$ & $C_{3}$ & $C_{4}$ & $C_{8}$ & $C_{4}$ & $C_{3}$ & $C_{4}$ & $C_{3}$ & $C_{8}$ & $C_{3}$ & $C_{8}$ & $C_{4}$ & $C_{4}$ & $C_{3}$ & $C_{8}$ \\
\hline
\end{tabular}

Figure 1. Random assignment of the levels of three factors in one block of the SSPD

We can notice that using the proposed construction method, the resulting incomplete SSPD has $b=36$ blocks with size equal to $k=18$ (three whole plots, 2 subplots and 3 sub-subplots). We also note that using the usual Kronecker product of matrices with $\otimes$, the incomplete SSPD would be 9 times larger (the number of blocks is then equal to 324 , and the number of experimental units $n=5832$ ). 
Table 6. Stratum efficiency factors of the SSPD in this example

\begin{tabular}{|c|c|c|c|c|c|c|}
\hline \multirow{2}{*}{\multicolumn{2}{|c|}{$\begin{array}{l}\text { Type of contrast } \\
\text { (No. of vectors) }\end{array}$}} & \multirow{3}{*}{$\frac{\mathrm{df}}{1}$} & \multicolumn{4}{|c|}{ Strata } \\
\hline & & & \multirow{2}{*}{$\frac{\mathrm{I}}{1 / 9}$} & \multirow{2}{*}{$\begin{array}{c}\text { II } \\
8 / 9\end{array}$} & \multirow{2}{*}{$\frac{\text { III }}{-}$} & \multirow{2}{*}{$\frac{\text { IV }}{-}$} \\
\hline$A$ & (2) & & & & & \\
\hline & (2) & 2 & $4 / 9$ & $5 / 9$ & - & - \\
\hline & (2) & 2 & - & 1 & - & - \\
\hline B & (8) & 3 & $1 / 3$ & - & $2 / 3$ & - \\
\hline \multirow[t]{2}{*}{$A \times B$} & (9) & 3 & $1 / 3$ & - & $2 / 3$ & - \\
\hline & (10) & 12 & - & $1 / 3$ & $2 / 3$ & - \\
\hline \multirow[t]{2}{*}{$C$} & (3) & 6 & $1 / 3$ & - & - & $2 / 3$ \\
\hline & (6) & 2 & - & - & - & 1 \\
\hline \multirow[t]{3}{*}{$A \times C$} & (4) & 6 & $1 / 3$ & - & - & $2 / 3$ \\
\hline & (5) & 24 & - & $1 / 3$ & - & $2 / 3$ \\
\hline & (7) & 10 & - & - & - & 1 \\
\hline \multirow[t]{3}{*}{$B \times C$} & (11) & 6 & $1 / 3$ & - & - & $2 / 3$ \\
\hline & (14) & 12 & - & - & $1 / 3$ & $2 / 3$ \\
\hline & (16) & 6 & - & - & - & 1 \\
\hline \multirow[t]{4}{*}{$A \times B \times C$} & (12) & 6 & $1 / 3$ & - & - & $2 / 3$ \\
\hline & (13) & 24 & - & $1 / 3$ & - & $2 / 3$ \\
\hline & (15) & 60 & - & - & $1 / 3$ & $2 / 3$ \\
\hline & (17) & 30 & - & - & - & 1 \\
\hline
\end{tabular}

The eigenvalues (efficiency factors) and eigenvectors of the constructed SSPD depend on the efficiency factors of the component designs. Hence, the statistical properties of the component designs used in this construction lead to efficiency factors of the final design (Table 6) which are different from the efficiency factors in Table 4.

Because of some incompleteness of the SSPD, we have lost a little efficiency for some treatment contrasts, and we have a loss of estimability of some treatment contrasts in some strata. The general hypothesis concerning the effects of main sources of variability in the experiment can also be tested using the full set of orthogonal basic contrasts. It is only necessary to have a stratum (or strata) in which all basic treatment contrasts are estimable.

In particular, the general hypothesis concerning main effects of the factor $A$ is testable in stratum II, while the hypotheses concerning the main effects of the factor $B$ and the interaction effects of $A \times B$ are testable in stratum III. The other hypotheses are testable in stratum IV. To improve the statistical properties of 
inference in the area of estimation, as well as in the area of hypothesis testing, some methods of combining information can be applied.

\section{Conclusion}

We have proposed a method of construction to obtain an incomplete split-splitplot design with very desirable statistical properties, especially the utilization of a small number of observations. As the main tools, resolvable block designs and the semi-Kronecker product of matrices are used. In particular, the generating incidence matrices for factors $A, B, C$, i.e. $\boldsymbol{N}_{A}, \boldsymbol{N}_{B}$ and $\boldsymbol{N}_{C}$, must have the same number of partitions to apply the semi-Kronecker product. Additionally, for the factor $A$ we can use any resolvable block design, while for factors $B$ and $C$ square lattice designs are used. This is a slight disadvantage of the method, because square lattice designs exist only for some sets of design parameters. In this example, for the factor $A$ with $v^{\prime}=6$ levels we use a resolvable block design. For the remaining factors there are square lattice designs, where for the factor $B$ it is a balanced square $\left(r=s_{1}+1=2+1=3\right)$, and for the factor $C$ it is not a balanced square $\left(r=3<s_{2}+1=4\right)$.

The main advantage of the construction method proposed is that the size of the final experiment with desirable statistical properties is small. The requirement for properties such as general balance or orthogonal block structure entails a larger number of blocks in the experiment, but the number is always smaller than in the traditional method where the usual Kronecker product of generating matrices is used. It is an obvious fact that in experiments, complete designs, which are orthogonal designs, are the best in terms of statistical properties. They make it possible to estimate with full efficiencies (efficiency factors equal to 1) all comparisons of the treatment effects. However, there are situations where they cannot always be applied. For example, for various reasons, there may be a smaller block size involved in an experiment relative to the number of treatment combinations. One of the solutions to the problem is to use some incomplete balanced and/or partially balanced designs in the experiment, like the incomplete 
SSPD proposed in this paper. Note that some treatment contrasts in the proposed design can be estimated with full efficiencies as in the complete (orthogonal) SSPD. Having the structure of the vectors (1)-(23) generating treatment contrasts, we can decide the level of the efficiency of estimation of particular treatment effects.

Acknowledgements: This work was supported in part by JSPS Grant-in-Aid for Scientific Research (C) 16K00053.

\section{REFERENCES}

Ambroży, K., Mejza, I. (2011): Statistical properties of some supplemented split-splitplot design. Colloquium Biometricum 41, 165-174.

Ambroży, K., Mejza, I. (2012): Control whole plot treatments in some split-split-plot experiment designs generated by orthogonally supplemented block design. Colloquium Biometricum 42, 77-84.

Ambroży, K., Mejza, I. (2013): A method of constructing incomplete split-split-plot designs supplemented by whole plot and subplot standards and their analysis. Colloquium Biometricum 43, 59-72.

Clatworthy, W.H. (1973): Tables of two associate class partially balanced designs. NBS App. Math. Ser. 63, Department of Commerce.

Gomez, K.A., Gomez, A.A. (1984): Statistical procedures for agricultural research. Wiley, New York.

Gupta, S., Mukerjee, R. (1989): A calculus for factorial arrangements. Lecture Notes in Statistics 59, Springer-Verlag.

Houtman, A.M., Speed, T.P. (1983): Balance in designed experiments with orthogonal block structure. The Annals of Statistics 11, 1069-1085.

Khatri, C.G., Rao, C.R. (1968): Solutions to some functional equations and their applications to characterization of probability distributions. Sankya, A, 30,167-180.

Kuriki, S., Nakajima, K. (2007): Square Lattice Designs in Incomplete Split-Plot Designs. Journal of Statistical Theory and Practice 1(3-4), 417-426.

Mejza, I. (1997a): Incomplete split-split-plot designs. 51st Session of the International Statistical Institute ISI'97, Istanbul. Contributed Papers, Book 1, 221-222.

Mejza, I. (1997b): Three factor experiments in incomplete split-split-plot designs. Colloquium Biometryczne 27, 127-138 (in Polish).

Mejza I., Kuriki S., Mejza S. (2001): Balanced square lattice designs in split-plot designs. Colloquium Biometryczne 31, 97-103.

Mejza, I., Mejza, S. (1984): Incomplete split plot designs. Statistics \& Probability Letters $2,327-332$.

Mejza, I., Mejza, S., Kuriki, S. (2012): A Method of Constructing Incomplete Split-Plot Designs Supplemented by Control Treatments and Their Analysis. Journal of Statistical Theory and Practice 6, 204-219. 
Mejza, S. (1992): On some aspects of general balance in designed experiments. Statistica 52, 263-278.

Nelder, J.A. (1965a): The analysis of randomized experiments with orthogonal block structure. 1. Block structure and the null analysis of variance. Proc. of the Royal Soc. of Lond. Ser. A, 283, 147-162.

Nelder, J.A. (1965b): The analysis of randomized experiments with orthogonal block structure. 2. Treatment structure and general analysis of variance. Proc. of the Royal Soc. of Lond. Ser. A, 283, 163-178.

Ozawa, K., Mejza, S., Jimbo, M., Mejza, I., Kuriki, S. (2004): Incomplete split-plot designs generated by some resolvable balanced designs. Statistics \& Probability Letters 68, 9-15.

Ozawa, K., Mejza, I., Mejza, S., Kuriki, S. (2018): Two-factor experiments with split units constructed by cyclic designs and square lattice designs. Revstat-Stat. J. 16(3), 279-294.

Raghavarao, D. (1971): Constructions and combinatorial problems in design of experiments. New York, John Wiley \& Sons.

Rao, C.R., Mitra, S.K. (1971): Generalized inverse of matrices and its applications. Wiley, New York.

Pearce, S.C., Caliński, T., Marshall, T.F. de C. (1974): The basic contrasts of an experimental design with special reference to the analysis of data. Biometrika 61, 449-460.

Whitaker, D., Williams, E.R., John, J.A. (2002): A package for the computer generation of experimental designs, CycDesigN Ver. 2.0. Canberra, Australia, CSIRO. 